\title{
Estimates for the Derivatives of the Poisson Kernel on Nilpotent Meta-Abelian Groups
}

\author{
Richard Penney • Roman Urban
}

Received: 12 March 2013 / Accepted: 15 August 2013 / Published online: 30 August 2013

(C) The Author(s) 2013. This article is published with open access at Springerlink.com

\begin{abstract}
Let $S$ be a semi direct product $S=N \rtimes A$ where $N$ is a connected and simply connected, non-abelian, nilpotent meta-abelian Lie group and $A$ is isomorphic with $\mathbb{R}^{k}, k>1$. We consider a class of second order left-invariant differential operators on $S$ of the form $\mathcal{L}_{\alpha}=L^{a}+\Delta_{\alpha}$, where $\alpha \in \mathbb{R}^{k}$, and for each $a \in \mathbb{R}^{k}, L^{a}$ is left-invariant second order differential operator on $N$ and $\Delta_{\alpha}=\Delta-\langle\alpha, \nabla\rangle$, where $\Delta$ is the usual Laplacian on $\mathbb{R}^{k}$. Using some probabilistic techniques (skew-product formulas for diffusions on $S$ and $N$ respectively, the concept of the derivative of a measure, etc.) we obtain an upper bound for the derivatives of the Poisson kernel for $\mathcal{L}_{\alpha}$. During the course of the proof we also get an upper estimate for the derivatives of the transition probabilities of the evolution on $N$ generated by $L^{\sigma(t)}$, where $\sigma$ is a continuous function from $[0, \infty)$ to $\mathbb{R}^{k}$.
\end{abstract}

Keywords Poisson kernel • Evolution kernel • Harmonic functions • Left invariant differential operators • Elliptic and parabolic equations • Evolution equation • Meta-Abelian nilpotent Lie groups • Solvable Lie groups • Homogeneous groups • Higher rank $N A$ groups • Brownian motion • Exponential functionals of Brownian motion

Mathematics Subject Classifications (2010) 58J05 • 35K10 • 43A85 • 31B05 - 22E25 • $22 \mathrm{E} 30 \cdot 60 \mathrm{~J} 25 \cdot 60 \mathrm{~J} 60$

\footnotetext{
R. Penney

Department of Mathematics, Purdue University, 150 N. University St, West Lafayette, IN 47907, USA

e-mail: rcp@math.purdue.edu

R. Urban $(\bowtie)$

Institute of Mathematics, Wroclaw University, Plac Grunwaldzki 2/4,

50-384 Wroclaw, Poland

e-mail: urban@math.uni.wroc.pl
} 


\section{Introduction}

Let $S$ be a semi direct product $S=N \rtimes A$ where $N$ is a connected, simply connected, non-abelian, nilpotent, meta-abelian, group and $A$ is isomorphic with $\mathbb{R}^{k}$. Specifically, we assume that

$$
N=M \rtimes V,
$$

where $M$ and $V$ are abelian Lie groups with the corresponding Lie algebras $\mathfrak{m}$ and $\mathfrak{v}$. Then there are bases $\left\{X_{1}, \ldots, X_{m}\right\}$ and $\left\{Y_{1}, \ldots, Y_{n}\right\}$ for $\mathfrak{m}$ and $\mathfrak{v}$ respectively such that $\left\{X_{1}, \ldots, X_{m}, Y_{1}, \ldots, Y_{n}\right\}$ forms a Jordan-Hölder basis for the Lie algebra $\mathfrak{n}$ of $N$ which diagonalizes the $\operatorname{ad}_{\mathfrak{a}}$ action on $\mathfrak{n}(\mathfrak{a}$ is the Lie algebra of $A$ ). We assume that these bases are ordered so that the matrix of $\operatorname{ad}_{Z}$ is strictly lower triangular for all $Z \in \mathfrak{n}$. We use these bases to identify $\mathfrak{m}$ and $\mathfrak{v}$ with $\mathbb{R}^{m}$ and $\mathbb{R}^{n}$ respectively and we use the exponential map to identify $M, V$ and $A$ with the corresponding Lie algebras.

In what follows the Euclidean space $\mathbb{R}^{k}$ is endowed with the usual scalar product $\langle\cdot, \cdot\rangle$ and the corresponding $\ell^{2}$ norm $\|\cdot\|$. For the vector $x \in \mathbb{R}^{k}$ we write $x^{2}=x \cdot x=$ $\langle x, x\rangle=\sum_{i=1}^{k} x_{i}^{2}$. By $\|\cdot\|_{\infty}$, we denote the $\ell^{\infty}$ norm $\|x\|_{\infty}=\max _{1 \leq i \leq k}\left|x_{i}\right|$.

For $g \in S$ we let $z(g)=z \in N$ and $a(g)=a \in A$ denote the components of $g$ in $N \rtimes A$ so that $g=(z, a)$. Similarly, for $z \in N$ we let $x(z)=x \in M$ and $y(z)=y \in V$ denote the components of $z$ in $M \rtimes V$. The dimension $k$ of $A$ is called the rank of $S$.

Let

$$
\begin{aligned}
& \Xi=\left\{\xi_{1}, \ldots, \xi_{m}\right\}, \\
& \Theta=\left\{\vartheta_{1}, \ldots, \vartheta_{n}\right\}
\end{aligned}
$$

be the roots of the $\operatorname{ad}_{\mathfrak{a}}$ action on $\mathfrak{m}$ and $\mathfrak{v}$ respectively corresponding to the given bases. Let

$$
\Lambda=\Xi \cup \Theta
$$

Hence, for all $H \in \mathfrak{a}$,

$$
\begin{aligned}
& \operatorname{ad}_{H} X_{i}=\left[H, X_{i}\right]=\xi_{i}(H) X_{i}, 1 \leq i \leq m, \\
& \operatorname{ad}_{H} Y_{j}=\left[H, Y_{j}\right]=\vartheta_{j}(H) Y_{j}, 1 \leq j \leq n .
\end{aligned}
$$

Let $q=m+n$. For $1 \leq i \leq q$ we set

$$
\lambda_{i}= \begin{cases}\xi_{i}, & 1 \leq i \leq m, \\ \vartheta_{i-m}, & m+1 \leq i \leq q .\end{cases}
$$

The principal object of study in this work is the left-invariant differential operator on $S$,

$$
\mathcal{L}_{\alpha}=\Delta_{\alpha}+\sum_{j=1}^{m} e^{2 \xi_{j}(a)} X_{j}^{2}+\sum_{j=1}^{n} e^{2 \vartheta_{j}(a)} Y_{j}^{2}
$$

\footnotetext{
${ }^{1}$ The case where $N$ is abelian can be analyzed by considerably simpler methods which we do not consider here.
} 
where, for $\alpha=\left(\alpha_{1}, \ldots, \alpha_{k}\right) \in \mathbb{R}^{k}$,

$$
\Delta_{\alpha}=\sum_{i=1}^{k}\left(\partial_{a_{i}}^{2}-2 \alpha_{i} \partial_{a_{i}}\right)
$$

and the $Y_{i}$ and $X_{j}$ are considered as left invariant differential operators on $N$. We assume that for all $i$,

$$
\lambda_{i}(\alpha)>0 .
$$

In particular, none of the $\lambda_{i}$ are identically 0 . Hence the $\left\{\lambda_{i}\right\}_{1 \leq i \leq q}$ span $\mathfrak{a}^{*}$ since their joint nullspace consists of vectors annihilated by $\mathrm{ad}_{\mathfrak{a}}$.

We set

$$
A^{+}=\operatorname{Int}\left\{a \in \mathbb{R}^{k}: \lambda_{i}(a) \geq 0 \text { for } 1 \leq i \leq q\right\} .
$$

Thus inequality (1.3) means that $\alpha \in A^{+}$.

We study the Poisson kernel for the operators (1.2). To describe this concept let $\chi$ be the modular function for left invariant Haar measure on $S$. Thus for all $g \in S$,

$$
\int_{S} f(s g) d s=\chi(g)^{-1} \int_{S} f(s) d s,
$$

where $d s$ is left-invariant Haar measure on $S$. Then

$$
\chi(g)=\operatorname{det}(\operatorname{Ad}(g))=e^{\rho(a)},
$$

where

$$
\rho=\sum_{j=1}^{q} \lambda_{j}
$$

Assumption (1.3) together with [5] implies there exists a Poisson kernel $v$ for $\mathcal{L}_{\alpha}$. That is, there is a $C^{\infty}$ function $v$ on $N$ such that every bounded $\mathcal{L}_{\alpha}$-harmonic function $F$ on $S$ may be written as a Poisson integral against a bounded function $f$ on the quotient space $A \backslash S=N$,

$$
F(g)=\int_{A \backslash S} f(g z) v(z) d z=\int_{N} f(z) \check{v}^{a}\left(z^{-1} z_{o}\right) d z, \quad g=\left(z_{o}, a_{o}\right),
$$

where

$$
\check{v}^{a}(z)=\check{v}\left(a^{-1} z a\right) \chi(a)^{-1} \text { and } \breve{v}(z)=v\left(z^{-1}\right) .
$$

Conversely the Poisson integral of any $f \in L^{\infty}(N)$ is a bounded $\mathcal{L}_{\alpha}$-harmonic function.

For $I=\left(i_{1}, \ldots, i_{m}\right) \in(\mathbb{N} \cup\{0\})^{m}$, let $|I|=i_{1}+\ldots+i_{m}$, and

$$
X^{I}=\prod_{j=1}^{m} X_{i}^{i_{j}}
$$

thought of as an element of the universal enveloping algebra $\mathfrak{A}(\mathfrak{m})$ of $\mathfrak{m}$. Similarly, for $J=\left(j_{1}, \ldots, j_{n}\right)$ we define $Y^{J} \in \mathfrak{A}(\mathfrak{v})$. 
Our goal in this work is to obtain growth estimates for the functions

$$
X^{I} Y^{J} v(z), \quad z \in N
$$

for general multi-indices $I$ and $J$ in the rank $S>1$ case. In the rank one case, the growth estimates for both of $v(z)$ and its derivatives are well understood, even for general nilpotent $N$, due to a number of works such as [4, 6-8, 13]. However, virtually nothing seems to be known about the growth estimates for the derivatives of the Poisson kernel in higher rank. The techniques used in the above mentioned works do not seem to generalize to higher rank groups, even for the $I=J=0$ case.

In $[11,12]$ the authors introduced some new techniques for studying the growth of the Poisson kernel in higher rank cases. At that time we had hoped that these techniques could finally yield insights into the growth of the derivatives of the Poisson kernel in the higher rank case. This hope is, in a sense, validated by the current work. However, the analysis of the growth of the derivatives, even given the work in [12], has forced the introduction of a host of new, and we feel exciting, techniques. (See Section 2 for an outline of some of these techniques.)

To describe our main result, we identify $\left(\mathbb{R}^{k}\right)^{*}$ with $\mathbb{R}^{k}$. This allows us to write

$$
\lambda_{i}=\left(\lambda_{i, 1}, \ldots, \lambda_{i, k}\right) .
$$

We say that positivity holds if all of the $\lambda_{i, j}$ are non-negative.

To state our main result we require some notation. If $\mathcal{F} \subset \Lambda$ is any set of roots and $a \in \mathbb{R}^{k}$, let

$$
a_{\mathcal{F}}^{\min }=\min _{\lambda \in \mathcal{F}}\left\{\lambda_{j}(a)\right\}, \quad a_{\mathcal{F}}^{\max }=\max _{\lambda \in \mathcal{F}}\left\{\lambda_{j}(a)\right\}, \quad \bar{a}_{\mathcal{F}}=\frac{a_{\mathcal{F}}^{\max }-a_{\mathcal{F}}^{\min }}{a_{\mathcal{F}}^{\min }},
$$

and

$$
\begin{aligned}
& \varkappa(I, J)=|J|+\bar{\rho}_{\Xi}(|I|+2|J|), \\
& \lambda(I, J)=\sum_{\ell=1}^{m} i_{\ell} \lambda_{\ell}+\sum_{\ell=1}^{n} j_{\ell} \lambda_{\ell+m} .
\end{aligned}
$$

Let $\mathcal{T}=\left\{\tau_{1}, \ldots, \tau_{\ell}\right\}$ be an orthogonal family of vectors in $\mathbb{R}^{k}$ such that $\alpha \cdot \tau_{i}>0$ for all $i$. Let

$$
d(\mathcal{T})=2^{-\ell+3} \sqrt{2} \min \left\{\frac{\alpha \cdot v}{\|\alpha\|\|v\|} \mid v=\tau_{i_{1}}+\cdots+\tau_{i_{j}}, 1 \leq i_{j} \leq \ell, 1 \leq j \leq \ell\right\} .
$$

We assume the positivity condition-i.e., the $\lambda_{i, j}$ in Eq. 1.7 are non-negative. Let

$$
d_{i}=d\left(\left\{\lambda_{i, 1} e_{1}, \ldots, \lambda_{i, k} e_{k}\right\} \backslash\{0\}\right), 1 \leq i \leq q,
$$

and define,

$$
d=\min _{1 \leq i \leq q} d_{i} / 2 .
$$

We also assume that for $1 \leq \ell \leq k$,

$$
\begin{aligned}
\alpha_{\ell} & >(\varkappa(I, J) / 2+|I|) \lambda_{i, \ell}, 1 \leq i \leq m, \text { and } \\
\alpha_{\ell} & >|J| \lambda_{j, \ell}, m+1 \leq j \leq q, \\
\|\alpha\| & >4 \max \{\varkappa(I, J), 2(|I|+|J|), 4 d\} / d .
\end{aligned}
$$


Theorem 1.1 Let $K \subset N=M \rtimes V$ be a compact set not containing the identity element $e \in N$. Suppose that the positivity condition below Eq. 1.7 holds for all $\lambda_{i}$, $1 \leq i \leq q$. Then, under the assumptions (1.3) and (1.8), there is a $C>0$ such that for all $z \in K$ and for all $a \in A^{+}$,

$$
\left|X^{I} Y^{J} v\left(a z a^{-1}\right)\right| \leq C e^{-a \cdot(\rho+\lambda(I, J))+a_{\Xi}^{\max }\left(1+\bar{\rho}_{\Xi} / 2\right)(|I|+|J|)},
$$

where $|I|+|J| \neq 0$.

For $t \in \mathbb{R}^{+}$and $a \in A^{+}$, let

$$
\delta_{t}^{a}=\left.\operatorname{Ad}((\log t) a)\right|_{N} .
$$

Then $t \mapsto \delta_{t}^{a}$ is a one parameter group of automorphisms of $N$ for which the corresponding eigenvalues on $\mathfrak{n}$ are all positive. It is known [9] that then $N$ has a $\delta_{t}^{a}$-homogeneous norm: a non-negative continuous function $|\cdot|_{a}$ on $N$ such that $|z|=0$ if and only if $z=e$ and

$$
\left|\delta_{t}^{a} z\right|_{a}=t|z|_{a}
$$

Corollary 1.2 Let $a \in A^{+}$be given. Then, under the assumptions of Theorem 1.1, for all $|z|_{a} \geq 1$,

$$
\left|X^{I} Y^{J} v(z)\right| \leq C|z|_{a}^{-a \cdot(\rho+\lambda(I, J))+a_{\Xi}^{\max }\left(1+\bar{\rho}_{\Xi} / 2\right)(|I|+|J|)} .
$$

\subsection{Example}

Consider $N=\mathcal{H}_{n}$, the $(2 n+1)$-dimensional Heisenberg group, which we realize as $\mathbb{R}^{n} \times \mathbb{R}^{n} \times \mathbb{R}$ with the Lie group multiplication given by

$$
\left(x_{1}, y_{1}, z_{1}\right)\left(x_{2}, y_{2}, z_{2}\right)=\left(x_{1}+x_{2}, y_{1}+y_{2}, z_{1}+z_{2}+x_{1} \cdot y_{2}\right),
$$

where $\cdot$ denotes the scalar product in $\mathbb{R}^{n}$.

The corresponding Lie algebra $\mathfrak{h}_{n}$ is then spanned by the left invariant vector fields

$$
X_{j}=\partial_{x_{j}}, \quad Y_{j}=\partial_{y_{j}}+x_{j} \partial_{z}, \quad 1 \leq j \leq n,
$$

and

$$
X_{n+1}=\partial_{z}
$$

which satisfy

$$
\left[X_{i}, Y_{j}\right]=\delta_{i, j} X_{n+1}, \quad 1 \leq i \leq n+1,1 \leq j \leq n .
$$

Let $\mathfrak{a}=\mathbb{R}^{n}$ and let $\left\{A_{1}, \ldots, A_{n}\right\}$ be the standard basis for $\mathbb{R}^{n}$ and let the corresponding dual basis for $\left(\mathbb{R}^{n}\right)^{*}$ be $\left\{e_{1}, \ldots, e_{n}\right\}$. We define an $\mathfrak{a}$ action on $\mathfrak{h}_{n}$, the Lie algebra of $\mathcal{H}_{n}$, by

$$
\begin{gathered}
{\left[A_{i}, X_{j}\right]=\delta_{i, j} X_{j}, \quad 1 \leq i, j \leq n,} \\
{\left[A_{i}, Y_{j}\right]=\delta_{i, j} Y_{j}, \quad 1 \leq i, j \leq n,} \\
{\left[A_{i}, X_{n+1}\right]=2 X_{n+1}, \quad 1 \leq i \leq n .}
\end{gathered}
$$


Exponentiation yields a group action of $A=\mathbb{R}^{n}$ on $\mathcal{H}_{n}$ and a solvable Lie group $S=\mathcal{H}_{n} \rtimes \mathbb{R}^{n}$. Then

$$
\begin{aligned}
\xi_{i} & =\vartheta_{i}=e_{i}, \quad 1 \leq i \leq n, \\
\xi_{n+1} & =2 \sum_{i=1}^{n} e_{i}=\rho / 2, \\
\lambda(I, J) & =I+J .
\end{aligned}
$$

It is clear that the positivity condition below Eq. 1.7 holds.

Let $\alpha=\left(\alpha_{1}, \ldots, \alpha_{n}\right)$ where $0<\alpha_{1} \leq \alpha_{2} \cdots \leq \alpha_{n}$. It is easily checked that

$$
d=\frac{d_{n+1}}{2}=d\left(\left\{2 e_{1}, \ldots, 2 e_{n}\right\}\right)=2^{-n+\frac{5}{2}} \frac{\alpha_{1}}{\|\alpha\|} .
$$

(Note that for any positive increasing sequence $\beta_{i}, \sqrt{\ell} \beta_{1} \leq \sum_{1}^{\ell} \beta_{i}$.)

Now let $a=t \rho$. Then $\bar{\rho}_{\Xi}=0$ and $a_{\Xi}^{\max }=4 t$. Hence

$$
-a \cdot(\lambda(I, J))+a_{\Xi}^{\max }\left(1+\bar{\rho}_{\Xi} / 2\right)(|I|+|J|)=0 .
$$

Thus Theorem 1.1 gives

$$
\left|X^{I} Y^{J} v\left(a h a^{-1}\right)\right| \leq C e^{-t\|\rho\|^{2}}=e^{-16 n t}, \quad h \in K \subset \mathcal{H}_{n},
$$

for all $\alpha$ satisfying Eq. 1.8.

Corollary 1.2 states that under the same assumptions for all $h \in \mathcal{H}_{n}$ with $|h|_{\rho} \geq 1$,

$$
\left|X^{I} Y^{J} v(h)\right|_{\rho} \leq C|h|_{\rho}^{-16 n} .
$$

We do not expect that this estimate is optimal since the rate of decay should depend on $\alpha, I$, and $J$.

\section{Outline of the Proofs of the Main Results}

In this section we introduce some notation and describe for the reader's convenience the main idea of the proofs of the results stated above.

Our proofs make use of a well known probabilistic formula for $v^{a}$ on a general $N A$ group. Specifically, the diffusion $\sigma(t)$ on $\mathbb{R}^{k}$ generated by $\Delta_{\alpha}$, is the $k$-dimensional Brownian motion with drift $-2 \alpha$, i.e., $\sigma(t)=b(t)-2 \alpha t$.

Let

$$
L^{\sigma, t}=\sum_{j=1}^{m} e^{2 \xi_{j}(\sigma(t))} X_{j}^{2}+\sum_{j=1}^{n} e^{2 \vartheta_{j}(\sigma(t))} Y_{j}^{2},
$$

thought of as a time dependent family of left invariant operators on $N$. This family gives rise to a diffusion which is described by a family of convolution kernels $P_{t, s}^{\sigma}(z)$, $s \leq t, \quad z \in N$, which satisfy the Chapman-Kolmogorov equations with respect to convolution on $N$. (See [12, Section 2.3].) We let $P_{t}^{\sigma}=P_{t, 0}^{\sigma}$. We also typically drop the interval $(0, t)$ in our notation so that, for example, the symbols $A_{V, j}^{\sigma}(0, t)$ and $\Lambda^{\eta}(0, t)$ introduced below will usually be denoted by $A_{V, j}^{\sigma}$ and $\Lambda^{\eta}$, respectively. 
It follows from formula (5.3) of [12] that

$$
v^{a}(z)=\lim _{t \rightarrow \infty} \mathbf{E}_{a} \check{P}_{t}^{\sigma}(z)
$$

where the expectation is with respect to the Wiener measure $\mathbf{W}_{a}$ on the set of continuous paths in $\mathbb{R}^{k}$ starting at $a$.

From Eqs. 1.1 and 1.6, for $z \in N$ and $a \in A$,

$$
\begin{aligned}
\left(X^{I} Y^{J} v\right)\left(a^{-1} z a\right) & =e^{a \cdot \lambda(I, J)}\left(X^{I} Y^{J} v^{a}\right)(z) \chi(a) \\
& =e^{a \cdot(\rho+\lambda(I, J))} \lim _{t \rightarrow \infty} \mathbf{E}_{a} X^{I} Y^{J} \check{P}_{t}^{\sigma}(z) .
\end{aligned}
$$

Corollary 2.1 For $z \in N$ and $a \in A^{+}$,

$$
\left|X^{I} Y^{J} v(z)\right| \leq|z|_{a}^{-a \cdot(\rho+\lambda(I, J))} \max _{\left\{\left|z_{o}\right|_{a}=1\right\}} \lim _{t \rightarrow \infty}\left|\mathbf{E}_{-\left(\log |z|_{a}\right) a} X^{I} Y^{J} \check{P}_{t}^{\sigma}\left(z_{o}\right)\right| .
$$

Proof Let $z_{o}=\delta_{|z|_{a}^{-1}}^{a} z$. Then $\left|z_{o}\right|_{a}=1$ and

$$
\begin{aligned}
\left|X^{I} Y^{J} v(z)\right| & =\left|X^{I} Y^{J} v\left(\delta_{|z|_{a}}^{a}\left(z_{o}\right)\right)\right| \\
& =e^{-\left(\log |z|_{a}\right) a \cdot(\rho+\lambda(I, J))}\left|X^{I} Y^{J} v^{-\left(\log |z|_{a}\right) a}\left(z_{o}\right)\right| \\
& =|z|_{a}^{-a \cdot(\rho+\lambda(I, J))} \lim _{t \rightarrow \infty}\left|\mathbf{E}_{-\left(\log |z|_{a}\right) a} X^{I} Y^{J} \check{P}_{t}^{\sigma}\left(z_{o}\right)\right| .
\end{aligned}
$$

To bound the expectation in Corollary 2.1, we make use of a formula that expresses $P_{t}^{\sigma}$ as a kind of skew product of kernels on $M$ and $V$. Specifically, the family of left-invariant, time dependent operators on $V$

$$
L^{V, \sigma, t}=\sum_{j=1}^{n} e^{2 \vartheta_{j}(\sigma(t))} Y_{j}^{2}
$$

gives rise to diffusion on $V$ in the same manner as $L^{\sigma, t}$ defines a diffusion on $N$. This diffusion may be described by a process $X_{t}$ with state space $\mathbb{R}^{n}$ and, for each starting point $a \in \mathbb{R}^{n}$, a probability measure $\mathbf{W}_{a}^{V, \sigma}$ on $C\left([0, \infty), \mathbb{R}^{n}\right)$ which may be explicitly computed since $V$ is abelian (see Proposition 3.1). More generally, for each $T>0$ we obtain a probability measure $\mathbf{W}_{a}^{V, \sigma, T}$ on $C\left([0, T], \mathbb{R}^{n}\right)$.

Similarly, for all $\eta \in C\left([0, \infty), \mathbb{R}^{m}\right)$, the family of time dependent operators

$$
L^{M, \eta, \sigma, t}=\sum_{j=1}^{m} e^{2 \xi_{j}(\sigma(t))}\left(\operatorname{Ad}(\eta(t)) Y_{j}\right)^{2}
$$

gives rise to a diffusion having transition probabilities described by convolution kernels $P_{t, s}^{M, \eta, \sigma}(x)$ on $M$ which again may be explicitly computed (see formula (4.2)). Corollary 3.6 of [11] implies that for all $\psi \in C_{c}(V), t \leq T$, and a.e. $\sigma$,

$$
\begin{aligned}
\int_{V} P_{t}^{\sigma}(x, y) \psi(y) d y & =\int P_{t}^{M, \eta, \sigma}(x) \psi(\eta(t)) \mathbf{W}_{0}^{V, \sigma}(d \eta) \\
& =\int P_{t}^{M, \eta, \sigma}(x) \psi(\eta(t)) \mathbf{W}_{0}^{V, \sigma, T}(d \eta) .
\end{aligned}
$$


More generally let $\tilde{X}_{i}$ denote $X_{i}$ considered as a right-invariant differential operator on $N$. Then for all multi-indices $I$, and $t \leq T$,

$$
\int_{V} \tilde{X}^{I} P_{t}^{\sigma}(x, y) \psi(y) d y=\int X^{I} P_{t}^{M, \eta, \sigma}(x) \psi(\eta(t)) \mathbf{W}_{0}^{V, \sigma, T}(d \eta) .
$$

(Note that since $M$ is abelian, on $M, X^{I}=\tilde{X}^{I}$.) We provide upper bounds on $X^{I} P_{t}^{M, \eta, \sigma}(x)$ in Section 4 .

For the operators $Y^{I}$ the situation is more complicated. Here we make use of the concept of the derivative of a measure $[2,10]$. Let $\mathcal{V}$ be a vector space with a $\sigma$-algebra $\mathcal{F}$ of subsets of $\mathcal{V}$ which is invariant with respect to the shifts along a given vector $h \in \mathcal{V}$, i.e., if $A \in \mathcal{F}$ then $A+t h \in \mathcal{F}$ for every $t \in \mathbb{R}$. In this case we define

$$
\partial_{h} \mu=\lim _{t \rightarrow 0} t^{-1}\left(\mu_{t h}-\mu\right)
$$

provided this exists in the weak topology. It follows almost by definition that if $f$ is a $C^{\infty}$ function on $\mathcal{V}$ then

$$
\int \partial_{h} f d \mu=-\int f d \partial_{h} \mu .
$$

In Eq. 2.6, we are integrating

$$
\phi(\eta)=\psi(\eta(t))
$$

against the measure

$$
\mu_{T}^{I}(\eta)=X^{I} P_{t}^{M, \eta, \sigma}(x) \mathbf{W}_{0}^{V, \sigma, T}(\eta) .
$$

Let

$$
\gamma_{i}(t)=A_{V, i}^{\sigma}(0, t) Y_{i}
$$

where

$$
A_{V, i}^{\sigma}(s, t)=\int_{s}^{t} e^{2 \vartheta_{i}(\sigma(u))} d u .
$$

Since

$$
\partial_{\gamma_{i}}(\phi(\eta))=A_{V, i}^{\sigma}(0, t)\left(Y_{i} \psi\right)(\eta(t))
$$

we see

$$
\begin{aligned}
\int_{V} Y_{i} \tilde{X}^{I} P_{t}^{\sigma}(x, y) \psi(y) d y & =-\int_{V} \tilde{X}^{I} P_{t}^{\sigma}(x, y) Y_{i} \psi(y) d y \\
& =-\left(A_{V, i}^{\sigma}\right)^{-1} \int \partial_{\gamma_{i}} \phi(\eta) d \mu_{T}^{I}(\eta) \\
& =\int \phi(\eta) D_{t, i}\left(d \mu_{T}^{I}(\eta)\right),
\end{aligned}
$$

where

$$
D_{t, i}=\left(A_{V, i}^{\sigma}\right)^{-1} \partial_{\gamma_{i}} .
$$


On the other hand

$$
D_{t, i} \mu_{T}^{I}(\eta)=\left(D_{t, i} X^{I} P_{t}^{M, \sigma, \eta}(x)\right) \mathbf{W}_{0}^{V, \sigma, T}(\eta)+X^{I} P_{t}^{M, \sigma, \eta}(x) D_{t, i}\left(\mathbf{W}_{0}^{V, \sigma, T}(\eta)\right) .
$$

In Section 5.2 we show:

Proposition 2.2 For all $T>0$ and $1 \leq i \leq n$, the measure $\mathbf{W}_{0}^{V, \sigma, T}(d \eta)$ is differentiable along $\gamma_{i}$ and

$$
\partial_{\gamma_{i}} \mathbf{W}_{0}^{V, \sigma, T}(d \eta)=\eta_{i}(T) \mathbf{W}_{0}^{V, \sigma, T}(d \eta)
$$

From this point on we assume that $T=t$. To consider $Y^{J}$, let $Q^{n}(y, z)$ be the rational function on $\mathbb{R}^{2}$ defined by

$$
Q^{n}(y, z)=z^{-n}\left(z \frac{d}{d y}+y\right)^{n}(1) .
$$

It follows from Proposition 2.2 and induction that for any multi-index $J=\left(i_{1}, \ldots, i_{n}\right)$ of length $n$,

$$
D_{t}^{J} \mathbf{W}_{0}^{V, \sigma, t}(d \eta)=Q^{J}\left(\eta(t), A_{V}^{\sigma}\right) \mathbf{W}_{0}^{V, \sigma, t}
$$

where $D_{t}^{J}$ and $Q^{J}(z, y)$ are defined by

$$
Q^{J}(y, z)=\prod_{j=1}^{n} Q^{i_{j}}\left(y_{j}, z_{j}\right), \quad D_{t}^{J}=\prod_{j=1}^{n}\left(D_{t, j}\right)^{i_{j}},
$$

and $A_{V}^{\sigma}=\left(A_{V, 1}^{\sigma}, \ldots, A_{V, n}^{\sigma}\right)$.

Then

$$
\begin{aligned}
\int_{V} Y^{J} \tilde{X}^{I} P^{\sigma_{t}}(x, y) \psi(y) d y= & \int \phi(\eta) D_{t}^{J}\left(d \mu_{t}^{I}(\eta)\right) \\
= & \sum_{R+S=J} C_{R, S} \int \phi(\eta)\left(D_{t}^{R} X^{I} P_{t}^{M, \sigma, \eta}(x)\right) \\
& \times Q^{S}\left(A_{V}^{\sigma}, \eta(t)\right) \mathbf{W}_{0}^{N, \sigma, t}(\eta) .
\end{aligned}
$$

We bound the integrands in Section 5.2 and then obtain an upper bound (Theorem 6.1) for

$$
\left|\tilde{X}^{I} Y^{J} P_{t}^{\sigma}(x, y)\right|
$$

by estimating the expectations (with respect to the distribution of $\eta$ ).

Finally, in Section 7, we use Eq. 2.1, to get the estimate for derivatives of the Poisson kernel $v$, that is we take the limit (as $t \rightarrow \infty$ ) of the expectation (with respect to $\sigma$ ) of the upper bound of the quantity in Eq. 2.9. 


\section{The Diffusion on $V$}

Let

$$
L^{t}=\frac{1}{2} \sum_{i, j=1}^{n} a_{i j}(t) \partial_{i} \partial_{j}
$$

be a differential operator on $C^{\infty}\left(\mathbb{R}^{n}\right)$, where $\partial_{i}=\partial_{x_{i}}$ and $a(t)=\left[a_{i j}(t)\right]$ is a symmetric, positive definite matrix with entries belonging to $C([0, \infty), \mathbb{R})$. Proposition 2.9 of [12] states that for such an operator the transition functions are given by convolution against

$$
P_{t, s}(x)=\mathcal{D}(A(s, t)) e^{-\frac{1}{2}\left(A(s, t)^{-1} x \cdot x\right)},
$$

where

$$
A(s, t)=\int_{s}^{t} a(u) d u,
$$

and, for an $n \times n$ invertible matrix $A$ we set

$$
\mathcal{D}(A)=(2 \pi)^{-\frac{n}{2}}(\operatorname{det} A)^{-\frac{1}{2}} .
$$

Specifically, if we choose a basis of $\mathbb{R}^{n}$ so that $Y_{i}$ corresponds with $\partial_{x_{i}}$ then for the operator (2.3) the functions corresponding to $a$ and $A$ are, respectively,

$$
\begin{aligned}
a_{V}^{\sigma}(t) & =2 \operatorname{diag}\left[e^{2 \vartheta_{1}(\sigma(t))}, \ldots, e^{2 \vartheta_{n}(\sigma(t))}\right], \\
A_{V}^{\sigma}(s, t) & =2 \operatorname{diag}\left[A_{V, 1}^{\sigma}(s, t), \ldots, A_{V, j}^{\sigma}(s, n)\right],
\end{aligned}
$$

where

$$
A_{V, j}^{\sigma}(s, t)=\int_{s}^{t} e^{2 \vartheta_{j}(\sigma(u))} d u .
$$

Hence, by Eq. 3.2 the corresponding transition probabilities $P_{t, s}^{V, \sigma}(x, d y)$ satisfy

$$
\begin{aligned}
P_{t, s}^{V, \sigma}(x, d y) & =(4 \pi)^{-\frac{n}{2}} \prod_{j=1}^{n}\left(A_{V, j}^{\sigma}(s, t)\right)^{-\frac{1}{2}} \exp \left(-\sum_{j=1}^{n} \frac{\left(x_{j}-y_{j}\right)^{2}}{4 A_{V, j}^{\sigma}(s, t)}\right) d y_{j} \\
& =\prod_{j=1}^{n} p_{A_{V, j}^{\sigma}(s, t)}\left(x_{j}, d y_{j}\right),
\end{aligned}
$$

where $p_{t}(x, y)$ is a classical Gaussian kernel,

$$
p_{t}(x, y)=\frac{1}{\sqrt{4 \pi t}} e^{-\frac{(x-y)^{2}}{4 t}} .
$$

The kernel $p_{t}(x, y)$ is the transition function for the one dimensional Brownian process. $^{2}$

${ }^{2}$ Our normalization of the Brownian motion $b(s)$ is different than that typically used by probabilists who tend to assume that $\operatorname{Var} b(s)=s$. 
Thus the process $\eta(t)$ generated by $L^{V, \sigma, t}$ has coordinates $\eta_{j}(t)$ which are independent Brownian motions with time changed according to the clock governed by $\sigma$.

We may use this observation to realize our process. Let $T>0$ and $\sigma$ be fixed. Let

$$
T_{j}=A_{V, j}^{\sigma}(0, T), 1 \leq j \leq n .
$$

Let $\mathbf{W}_{0}^{T}$ be the product Wiener measure on the space

$$
\mathcal{V}_{1}^{T}=\prod_{j=1}^{n} C\left(\left[0, T_{j}\right], \mathbb{R}\right)
$$

i.e.,

$$
\mathbf{W}_{0}^{T}=\mathbf{W}_{0}^{T_{1}, \mathbb{R}} \otimes \ldots \otimes \mathbf{W}_{0}^{T_{n}, \mathbb{R}}
$$

where $\mathbf{W}_{0}^{T_{j}, \mathbb{R}}$ is the Wiener measure on $C\left(\left[0, T_{j}\right], \mathbb{R}\right)$, and let

$$
\mathcal{V}_{2}^{T}=(C([0, T], \mathbb{R}))^{n}=C\left([0, T], \mathbb{R}^{n}\right) .
$$

Consider the linear map

$$
\mathcal{T}^{T}: \mathcal{V}_{1}^{T} \rightarrow \mathcal{V}_{2}^{T}
$$

given by formula,

$$
\mathcal{T}^{T}(\eta)(u)=\left(\eta_{1}\left(A_{V, 1}^{\sigma}(0, u)\right), \ldots, \eta_{n}\left(A_{V, n}^{\sigma}(0, u)\right)\right), \quad u \in[0, T] .
$$

The following proposition is clear:

Proposition 3.1 The diffusion defined by $L^{V, \sigma, t}$ starting at $0,0 \leq t \leq T$ is realizable as the process $b_{t}$ on $\mathcal{V}_{1}^{T}$ with the probability measure

$$
\mathbf{W}_{0}^{V, \sigma, T}=\mathcal{T}^{T}\left(\mathbf{W}_{0}^{T}\right)
$$

We may of course apply the same ideas with the intervals $[0, T]$ and $\left[0, T_{j}\right]$ replaced by $[0, \infty)$ and $\left[0, T_{j}\right)$ respectively. In this case we omit the superscript $T$.

\section{The Diffusion on $M$}

From Section 3.1 of [12], the matrix $\left[a_{i j}\right]$ from Eq. 3.1 for the operator defined in Eq. 2.4 is

$$
\left[a_{M}^{\sigma, \eta}(t)\right]=2\left[\operatorname{Ad}(\eta(t)) S^{\sigma}(t)\right]\left[\operatorname{Ad}(\eta(t)) S^{\sigma}(t)\right]^{*},
$$

where

$$
S^{\sigma}(t)=\operatorname{diag}\left[e^{\xi_{1}(\sigma(t))}, \ldots, e^{\xi_{m}(\sigma(t))}\right]
$$

and the adjoint is in the $y_{j}$ coordinates.

Then from Eq. 3.2 the transition kernel $P_{t}^{M, \sigma, \eta}$ for the operator (2.4) satisfies

$$
P_{t}^{M, \sigma, \eta}(x)=\mathcal{D}\left(A_{M}^{\sigma, \eta}\right) \exp \left(-\frac{1}{2}\left(A_{M}^{\sigma, \eta}\right)^{-1} x \cdot x\right),
$$

where $A_{M}^{\sigma, \eta}=A_{M}^{\sigma, \eta}(0, t)$ is defined by Eq. 3.3. Recall that $P_{t}^{M, \sigma, \eta}$ denotes $P_{t, 0}^{M, \sigma, \eta}$. 
Let

$$
\Lambda^{\eta}(s, t)=\sup _{s \leq u \leq t}\|\eta(u)\|_{\infty},
$$

where $\|\cdot\|_{\infty}$ is the $\ell^{\infty}$-norm on $\mathbb{R}^{n}$. We typically denote $\Lambda^{\eta}(0, t)$ by $\Lambda^{\eta}$.

We set

$$
\mathcal{C}(\sigma)=\mathcal{C}(\sigma)(t)=\left\|\left(A_{M}^{\sigma, 0}(0, t)\right)^{-1}\right\|\left\|A_{M}^{\sigma, 0}(0, t)\right\| .
$$

Let $\|\cdot\|$ be any norm on the set of $m \times m$ matrices. We need an estimate describing how $\left\|\left(A_{M}^{\sigma, \eta}\right)^{-1}\right\|$ depends on $\Lambda^{\eta}$.

Proposition 4.1 There is a $C>0$ such that

$$
\left\|\left(A_{M}^{\sigma, \eta}\right)^{-1}\right\| \leq C\left(1+\Lambda^{\eta}\right)^{\bar{\rho}_{\Xi}}(1+\mathcal{C}(\sigma))^{\bar{\rho}_{\Xi}}\left\|\left(A_{M}^{\sigma, 0}\right)^{-1}\right\| .
$$

Proof We let $C$ denote a generic constant depending only on $m$ that can change from line to line.

For $Y \in \mathfrak{v}$ let

$$
N(Y)=\left.\operatorname{Ad}_{Y}\right|_{\mathfrak{m}}-I_{\mathfrak{m}}
$$

Since

$$
\left.\operatorname{Ad}_{Y}\right|_{\mathfrak{m}}=\sum_{j=0}^{k_{o}} \frac{\left(\left.\operatorname{ad}_{Y}\right|_{\mathfrak{m}}\right)^{j}}{j !}
$$

we see that

$$
\|N(Y)\| \leq C\|Y\|(1+\|Y\|)^{k_{o}-1} .
$$

Let $\Lambda^{\eta}=\Lambda^{\eta}(0, t)=\ell$. Then for $0 \leq u \leq t$,

$$
\begin{aligned}
a_{M}^{\sigma, \eta}(u)= & (I+N(\eta)) \operatorname{Ad}(\sigma(u))^{2}(I+N(\eta))^{*}, \\
a_{M}^{\sigma, \eta}(u)-a_{M}^{\sigma, 0}(u)= & N(\eta) \operatorname{Ad}(\sigma(u))^{2} N(\eta)^{*}+N(\eta) \operatorname{Ad}(\sigma(u))^{2} \\
& +\operatorname{Ad}(\sigma(u))^{2} N(\eta)^{*}, \\
\left\|A_{M}^{\sigma, \eta}-A_{M}^{\sigma, 0}\right\| \leq & C\left(\ell+\ell^{2}\right)(1+\ell)^{2 k_{o}-2}\left\|A_{M}^{\sigma, 0}\right\| .
\end{aligned}
$$

Thus

$$
\begin{aligned}
\left\|I-\left(A_{M}^{\sigma, 0}\right)^{-1} A_{M}^{\sigma, \eta}\right\| & =\left\|\left(A_{M}^{\sigma, 0}\right)^{-1}\left(A_{M}^{\sigma, 0}-A_{M}^{\sigma, \eta}\right)\right\| \\
& \leq C \ell(1+\ell)^{2 k_{o}-1} \mathcal{C}(\sigma) .
\end{aligned}
$$

But

$$
\Lambda^{\eta^{-p \rho}} \leq \ell e^{-p \rho_{\mathrm{m}}^{\min }}
$$

$\left(\eta^{-p \rho}\right.$ denotes the action of $-p \rho$ on $\left.\eta\right)$. Choose $p>0$ so that

$$
C e^{-p \rho_{\Xi}^{\min }} \ell\left(1+e^{-p \rho_{\Xi}^{\min }} \ell\right)^{2 k_{o}}(\mathcal{C}(\sigma)+1)=1 / 2,
$$


where $C$ is as in the last line of Eq. 4.4. It follows from the series expansion of $(I-Y)^{-1}$ that

$$
\left\|\left(A_{M}^{\sigma, \eta^{-p \bar{\rho} \Xi}}\right)^{-1} A_{M}^{\sigma, 0}\right\| \leq C^{\prime}
$$

But

$$
\left(A_{M}^{\sigma, \eta}\right)^{-1} A_{M}^{\sigma, 0}=\operatorname{Ad}(p \rho)\left(A_{M}^{\sigma, \eta^{-p \rho}}\right)^{-1} A_{M}^{\sigma, 0} \operatorname{Ad}(-p \rho) .
$$

Hence

$$
\left\|\left(A_{M}^{\sigma, \eta}\right)^{-1} A^{\sigma, 0}\right\| \leq C^{\prime} e^{p\left(\rho_{\Xi}^{\max }-\rho_{\Xi}^{\min }\right)}=C^{\prime}\left(e^{p \rho_{\Xi}^{\min }}\right)^{\bar{\rho}_{\Xi}} .
$$

However, from Eq. 4.5,

$$
e^{p \rho_{\Xi}^{\min }} \leq 2^{2 k_{o}} C(\mathcal{C}(\sigma)+1) \ell
$$

from which our result follows.

Lemma 3.3 of [12] implies the following result:

Lemma 4.2 There is a constant $C>0$ such that

$$
\mathcal{D}\left(A_{M}^{\sigma, \eta}\right) \leq C \mathcal{D}\left(A_{M}^{\sigma, 0}\right) .
$$

We note also the following result that is an immediate consequence of Eq. 4.3:

Lemma 4.3 There exists a constant $C>0$ such that

$$
\left\|A_{M}^{\sigma, \eta}\right\| \leq C\left(1+\Lambda^{\eta}\right)^{2 k_{o}}\left\|A_{M}^{\sigma, 0}\right\| .
$$

\section{The Derivatives of $\boldsymbol{P}^{\sigma}$}

In this section we estimate the derivatives of the evolution kernel $P^{\sigma}$ described in formula (2.5). Let $A^{\sigma}$ be the $q \times q$ matrix

$$
A^{\sigma}=\left[\begin{array}{cc}
A_{M}^{\sigma, 0} & 0 \\
0 & A_{V}^{\sigma}
\end{array}\right] .
$$

We define

$$
\mathcal{D}\left(A^{\sigma}\right)=\mathcal{D}\left(A_{M}^{\sigma, 0}\right) \mathcal{D}\left(A_{V}^{\sigma}\right)
$$

For $0 \neq y \in \mathbb{R}^{n}$ given and $\varepsilon>0$, let ${ }^{3}$

$$
\psi_{\varepsilon}(\cdot)=\varepsilon^{-n} \mathbf{1}_{B_{\varepsilon}(y)}(\cdot),
$$

\footnotetext{
${ }^{3}$ By $\mathbf{1}_{A}$ we denote the characteristic function (or indicator function) of a given set $A$, i.e., $\mathbf{1}_{A}(x)=1$
} if $x \in A$ and is zero otherwise. 
where

$$
B_{\varepsilon}(y)=\prod_{j=1}^{n} B_{\varepsilon}^{1}\left(y_{j}\right) \text { and } B_{\varepsilon}^{1}\left(y_{j}\right)=\left[y_{j}-\varepsilon / 2, y_{j}+\varepsilon / 2\right] \text {. }
$$

Our estimates all follow from Theorem 5.5 which is a corollary of Proposition 5.1 below. Let, for $a \geq 0$,

$$
\begin{aligned}
\tilde{P}_{t}^{\sigma, \eta, a}(x) & =\mathcal{D}\left(A_{M}^{\sigma, 0}\right)\left(1+\Lambda^{\eta}\right)^{a} \exp \left(-\frac{D^{\prime}\|x\|^{2}}{\left(1+\Lambda^{\eta}\right)^{2 k_{o}}\left\|A_{M}^{\sigma, 0}\right\|}\right), \\
\tilde{P}_{t}^{\sigma, a}(x, y) & =\limsup _{\varepsilon \rightarrow 0}\left(\mathbf{E}_{0}^{\eta} \tilde{P}_{t}^{\sigma, \eta, a}(x) \psi_{\varepsilon}\left(\eta_{t}\right)\right),
\end{aligned}
$$

where $\mathbf{E}_{v}^{\eta}$ denotes expectation with respect to $\mathbf{W}_{v}^{V, \sigma}(d \eta)$. For $k \in \mathbb{N}, x \in M$, and $y \in V$ we let

$$
\begin{aligned}
\phi_{k}(x) & =\left(\frac{\|x\|^{1 /(k+1)}}{\|x\|^{1 /(k+1)}+1}\right)^{2 k}, \\
|(x, y)|_{k} & =\|x\|^{1 /(k+1)}+\|y\| .
\end{aligned}
$$

Proposition 5.1 For $a \geq 0$ given, there are positive constants $C, D$ such that

$$
\tilde{P}_{t}^{\sigma, a}(x, y) \leq C B_{t}^{\sigma, a}(x, y)\left(F_{1}+F_{2}\right) \exp \left(-\frac{D\|y\|^{2}}{\left\|A_{V}^{\sigma}\right\|}\right),
$$

where

$$
\begin{aligned}
B_{t}^{\sigma, a}(x, y) & =\mathcal{D}\left(A^{\sigma}\right)\left(1+|(x, y)|_{k_{o}}\right)^{a}\left(1+\left\|A_{V}^{\sigma}\right\|\right)^{(a+1) / 2}, \\
F_{1} & =\exp \left(-\frac{D\|x\|^{2}}{\left(|(x, y)|_{k_{o}}+1\right)^{2 k_{o}}\left\|A_{M}^{\sigma, 0}\right\|}\right), \\
F_{2} & =\exp \left(-\frac{D\|x\|^{2 /\left(k_{o}+1\right)}}{\left\|A_{V}^{\sigma}\right\|}\right) .
\end{aligned}
$$

Proof For $k \in \mathbb{N}$ and $\varepsilon>0$, we let

$$
\mathcal{A}_{k}=\left\{\eta \in C([0, \infty), M) \mid k-1 \leq \Lambda^{\eta}(0, t)<k\right\}
$$

and

$$
\mathcal{S}_{\varepsilon}=\operatorname{supp} \psi_{\varepsilon}\left(\eta_{t}\right)
$$

Note that

$$
\eta \in \mathcal{S}_{\varepsilon} \cap \mathcal{A}_{k} \Rightarrow k \geq\|\eta(t)\|_{\infty} \geq\|y\|_{\infty}-\varepsilon / 2 .
$$

It follows that

$$
\tilde{P}_{t}^{\sigma, a}(x, y) \leq C \mathcal{D}\left(A_{M}^{\sigma, 0}\right) \sum_{k \geq\|y\|_{\infty}} k^{a} \exp \left(-\frac{D\|x\|^{2}}{k^{2 k_{o}}}\right) \mathcal{E}_{k},
$$


where

$$
\mathcal{E}_{k}=\limsup _{\varepsilon \rightarrow 0^{+}} \varepsilon^{-n}\left(\mathbf{W}_{0}^{V, \sigma}\left(\mathcal{S}_{\varepsilon} \cap \mathcal{A}_{k}\right)\right) .
$$

(See Lemma 4.3 of [12].)

Lemma 4.4 of [12], together with the reasoning above formula (4.7) of [12], implies:

Lemma 5.2 Let $n_{o}$ be the smallest integer such that $n_{o} \geq\|y\|_{\infty}$. There are constants $C, D>0$ such that

$$
\mathcal{E}_{k} \leq C \mathcal{D}\left(A_{N}^{\sigma}\right) \exp \left(-\frac{D\|y\|^{2}}{\left\|A_{V}^{\sigma}\right\|}\right) \mathcal{F}_{k}
$$

where

$$
\mathcal{F}_{k}= \begin{cases}1 & \text { for } k=n_{o}, \\ \exp \left(-\left(2\left\|A_{V}^{\sigma}\right\|\right)^{-1}\left((k-1)-\|y\|_{\infty}\right)^{2}\right) & \text { for } k>n_{o}\end{cases}
$$

The first term in Eq. 5.4 is dominated by

$$
C \mathcal{D}\left(A_{M}^{\sigma, 0}\right)(1+\|y\|)^{a} \mathcal{E}_{n_{o}}
$$

which in turn is dominated by the term in Eq. 5.3 involving $F_{1}$.

The $F_{2}$ term comes from the following lemma upon setting $c=\|x\|^{1 /\left(k_{o}+1\right)}$ and $E=\left(2\left\|A_{V}^{\sigma}\right\|\right)^{-1}$.

Lemma 5.3 Let $D, E$, and $a \geq 0$ be given. Then there is $a C>0$, independent of $D, E$, and $a$ such that for all $c \geq 0$,

$$
\begin{aligned}
\sum_{k \geq\|y\|_{\infty}+1} k^{a} \exp \left(-\frac{D}{k^{2 k_{o}}}-E\left(k-\left(\|y\|_{\infty}+1\right)\right)^{2}\right) \\
\leq(c+1)\left(\|y\|_{\infty}+a^{\frac{1}{2}} E^{-\frac{1}{2}}+1\right)^{a} \exp \left(-\frac{D}{\left(\|y\|_{\infty}+c+1\right)^{2 k_{o}}}\right) \\
+C\left(\|y\|_{\infty}+a^{\frac{1}{2}} E^{-\frac{1}{2}}+1\right)^{a} E^{-\frac{1}{2}} \exp \left(-E c^{2} / 2\right) .
\end{aligned}
$$

Proof We first note the following lemma which is a simple calculus exercise.

Lemma 5.4 For all $x \geq b \geq 0$ and $a>0$,

$$
x^{a} e^{-(x-b)^{2}} \leq(b+\sqrt{a / 2})^{a} .
$$

We apply this lemma with $a:=2 a / E$, and raise the resulting inequality to the $\frac{E}{2}$ th power, concluding that the term on the left in Eq. 5.5 is dominated by

$$
\left(a^{\frac{1}{2}} E^{-\frac{1}{2}}+\|y\|_{\infty}+1\right)^{a} \sum_{k \geq\|y\|_{\infty}+1} \exp \left(-\frac{D}{k^{2 k_{o}}}-\frac{E}{2}\left(k-\|y\|_{\infty}-1\right)^{2}\right) .
$$


We split the sum in Eq. 5.6 into two parts corresponding to:

$$
\|y\|_{\infty}+1 \leq k \leq\|y\|_{\infty}+1+c \text { and } k>\|y\|_{\infty}+1+c .
$$

The first part is dominated by the first term on the right in Eq. 5.5. The second summation is dominated by

$$
\begin{aligned}
\int_{c}^{\infty} \exp \left(-E u^{2} / 2\right) d u & =C E^{-\frac{1}{2}} \int_{c \sqrt{E / 2}}^{\infty} \exp \left(-v^{2}\right) d v \\
& \leq C E^{-\frac{1}{2}} \exp \left(-E c^{2} / 2\right) .
\end{aligned}
$$

Our lemma follows.

Thus, Proposition 5.1 is proved.

Now let $\phi_{k}$ be as in Eq. 5.2. The main result of this section is:

Theorem 5.5 There are $C, D>0$ such that

$$
\tilde{P}_{t}^{\sigma, a}(x, y) \leq C B_{t}^{\sigma, a}(x, y) \exp \left(-\frac{D\|x\|^{2 /\left(k_{o}+1\right)} \phi_{k}(x)}{\left\|A^{\sigma}\right\|}-\frac{D\|y\|^{2}}{\left\|A_{V}^{\sigma}\right\|}\right) .
$$

Proof We note that

$$
\begin{aligned}
\frac{\|x\|^{2}}{\left(\|x\|^{1 /\left(k_{o}+1\right)}+1\right)^{2 k_{o}}} & =\|x\|^{2-\frac{2 k_{o}}{k_{o}+1}}\left(\frac{\|x\|^{1 /\left(k_{o}+1\right)}}{\|x\|^{1 /\left(k_{o}+1\right)}+1}\right)^{2 k_{o}} \\
& =\|x\|^{\frac{2}{k_{o}+1}} \phi_{k_{o}}(x) \leq\|x\|^{\frac{2}{k_{o}+1}} .
\end{aligned}
$$

Thus the term in Eq. 5.3 coming from $F_{2}$ is bounded by the right side of Eq. 5.7.

In the region $\|y\| \geq\|x\|^{\frac{1}{k_{o}+1}}$, the result follows from the observation that

$$
\frac{D\|y\|^{2}}{\left\|A_{V}^{\sigma}\right\|} \geq \frac{D\|x\|^{\frac{2}{k_{o}+1}}}{2\left\|A_{V}^{\sigma}\right\|}+\frac{D\|y\|^{2}}{2\left\|A_{V}^{\sigma}\right\|}
$$

while in the region $\|y\| \leq\|x\|^{\frac{1}{k_{o}+1}}$,

$$
|(x, y)|_{k_{o}} \leq 2\left(1+\|x\|^{\frac{1}{k_{o}+1}}\right)
$$

and the result again follows.

5.1 Derivatives of $P^{M, \sigma, \eta}(x)$ with Respect to $x$

For an $m \times m$ symmetric matrix $B$ let

$$
f_{B}(x)=\exp \left(-\frac{1}{2}(B x) \cdot x\right) \text {. }
$$

Then for $1 \leq i, j \leq m$,

$$
\begin{aligned}
X_{i} f_{B}(x) & =-\lambda_{i}(x) f_{B}(x) \text { and } \\
X_{j} X_{i} f_{B}(x) & =\left(\lambda_{j}(x) \lambda_{i}(x)-\beta_{i, j}\right) f_{B}(x),
\end{aligned}
$$


where

$$
\begin{aligned}
\lambda_{i}(x) & =\left(B X_{i}\right) \cdot x \text { and } \\
\beta_{i, j} & =\left(B X_{i}\right) \cdot\left(B X_{j}\right) .
\end{aligned}
$$

In general

$$
X^{I} f_{B}(x)=Q_{I} f_{B}(x),
$$

where $Q^{I}$ is a polynomial in the variables $\lambda_{i}(x), 1 \leq i \leq m$, and $\beta_{j, k}, 1 \leq j, k \leq m$. Furthermore, it is easily seen that

$$
\left|Q_{I}\right| \leq C(1+\|x\|)^{|I|}\|B\|^{|I|} .
$$

Hence, from Proposition 4.1,

Corollary 5.6 There is a $C>0$ such that

$$
X^{I} P_{t}^{M, \sigma, \eta}(x) \leq C(1+\|x\|)^{|I|}(\mathcal{C}(\sigma)+1)^{\bar{\rho}_{\Xi}|I|}\left\|\left(A_{M}^{\sigma, 0}\right)^{-1}\right\|^{|I|} \tilde{P}_{t}^{\sigma, \eta, \bar{\rho}_{\Xi}|I|}(x),
$$

where notation is as in Eq. 5.1.

The desired estimate on the function $X^{I} P_{t}^{\sigma}(x, y)$ follows immediately from Theorem 5.5.

5.2 Derivatives of $X^{I} P^{M, \sigma, \eta}(x)$ with Respect to $\eta$

As mentioned in Section 2, we also require estimates on the derivatives in $\eta$ along the curves $\gamma_{i}$ in Eq. 2.7 of the expression in equality (4.2). Our first result is:

Proposition 5.7 Let $D_{t}^{J}$ be as in Eq. 2.8 and $\rho \in A^{+}$. Then there is a $C>0$ such that

$$
\begin{aligned}
\left|D_{t}^{J} X^{I} P_{t}^{M, \sigma, \eta}(x)\right| \leq & (\mathcal{C}(\sigma)+1)^{\varkappa(I, J)}\left\|\left(A_{M}^{\sigma, 0}\right)^{-1}\right\|^{|I|}\left(1+\left\|\left(A_{M}^{\sigma, 0}\right)^{-1}\right\|\right)^{|J|} \\
& \times(1+\|x\|)^{|I|+2|J|} \tilde{P}_{t}^{\sigma, \eta, q(I, J)}(x),
\end{aligned}
$$

where notation is as in Eq. 5.1.

Proof From Eq. 4.1, since $V$ is abelian and $\gamma_{i}=A_{V, i}^{\sigma}$,

$$
\begin{aligned}
A_{M}^{\sigma, \eta+u \gamma_{i}} & =\int_{0}^{t} e^{\operatorname{ad}\left(u \gamma_{i}(v)\right)} a_{M}^{\sigma, \eta}(v)\left(e^{u \operatorname{ad}\left(\gamma_{i}(v)\right)}\right)^{*} d v, \\
D_{t, i} A_{M}^{\sigma, \eta} & =\operatorname{ad}\left(Y_{i}\right) A_{M, i}^{\sigma, \eta}+A_{M, i}^{\sigma, \eta}\left(\operatorname{ad}\left(Y_{i}\right)\right)^{*},
\end{aligned}
$$

where

$$
A_{M, i}^{\sigma, \eta}=\left(A_{V, i}^{\sigma}\right)^{-1} \int_{0}^{t} \gamma_{i}(u) a_{M}^{\sigma, \eta}(u) d u .
$$

In particular, since $\gamma_{i}(t)$ is increasing,

$$
\left\|D_{t, i} A_{M}^{\sigma, \eta}\right\| \leq C\left\|A_{M}^{\sigma, \eta}\right\| \leq C\left(1+\Lambda^{\eta}\right)\left\|A_{M}^{\sigma, 0}\right\| .
$$


More generally, it follows by induction that

$$
\left\|D_{t}^{J} A_{M}^{\sigma, \eta}\right\| \leq C\left(1+\Lambda^{\eta}\right)\left\|A_{M}^{\sigma, 0}\right\| .
$$

Differentiating Eq. 4.2 in $\eta$ is complicated by the $\left(A_{M}^{\sigma, \eta}\right)^{-1}$ term. However, from the proof of Proposition 2.9 of [12], the Fourier transform of $P_{t}^{M, \sigma, \eta}$ is

$$
\left(P_{t}^{M, \sigma, \eta}\right) \wedge(\xi)=\exp \left(-\frac{1}{2} A_{M}^{\sigma, \eta} \xi \cdot \xi\right) .
$$

Of course

$$
D_{t, i}\left(P_{t}^{M, \sigma, \eta}\right)^{\wedge}(\xi)=-\frac{1}{2}\left[\left(D_{t, i} A_{M}^{\sigma, \eta}\right) \xi \cdot \xi\right] \exp \left(-\frac{1}{2} A_{M}^{\sigma, \eta} \xi \cdot \xi\right) .
$$

By a decomposition of the multi-index $J$ we mean a finite set $\mathcal{P}$ of multi-indices such that

$$
J=\sum_{\tilde{J} \in \mathcal{P}} \tilde{J} .
$$

Let $\mathcal{P}_{J}$ be the set of all decompositions of $J$. It follows by induction that

$$
D_{t}^{J}\left(P^{M, \sigma, \eta}\right)^{\wedge}(\xi)=\sum_{\mathcal{J} \in \mathcal{P}_{J}} C_{\mathcal{J}}\left(\prod_{\tilde{J} \in \mathcal{J}}\left[\left(D_{t}^{\tilde{J}} A_{M}^{\sigma, \eta}\right) \xi \cdot \xi\right]\right) \exp \left(-\frac{1}{2} A_{M}^{\sigma, \eta} \xi \cdot \xi\right),
$$

where the $C_{\mathcal{J}}$ are constants indexed by $\mathcal{P}_{J}$. Forming the inverse Fourier transformation shows that $D_{t}^{J} P^{M, \sigma, \eta}(x)$ is a linear combination of terms of the form

$$
\left(\prod_{\tilde{J} \in \mathcal{J}}\left[\sum_{1 \leq i, j \leq m}\left(D_{t}^{\tilde{J}} A_{M}^{\sigma, \eta}\right)_{i, j} X_{i} X_{j}\right]\right) P^{M, \sigma, \eta}(x)
$$

where $\mathcal{J} \in \mathcal{P}_{J}$.

Let $\mathcal{J}=\left\{J_{1}, \ldots, J_{\ell}\right\}$. Expanding this product and multiplying by $X^{I}$ shows that $D_{t}^{J} X^{I} P^{M, \sigma, \eta}$ is a linear combination of terms of the form

$$
\left(\prod_{k=1}^{\ell}\left(D_{t}^{J_{j}} A_{M}^{\sigma, \eta}\right)_{i_{k}, j_{k}}\right)\left(\prod_{k=1}^{\ell} X_{i_{k}} X_{j_{k}}\right) X^{I} P^{M, \sigma, \eta},
$$

where $1 \leq i_{k}, j_{k} \leq m$.

From Corollary 5.6 the above expression is bounded by a multiple of

$$
\begin{aligned}
(1+ & \left.\Lambda^{\eta}\right)^{\ell}\left\|A_{M}^{\sigma, 0}\right\|^{\ell}\left\|\left(A_{M}^{\sigma, 0}\right)^{-1}\right\|^{|I|+2 \ell}(\mathcal{C}(\sigma)+1)^{\bar{\rho}_{\Xi}(|I|+2 \ell)} \\
& \times(1+\|x\|)^{|I|+2 \ell} \tilde{P}_{t}^{\sigma, \eta, \bar{\rho}_{\Xi}(|I|+2 \ell)}(x) .
\end{aligned}
$$

We note that

$$
\begin{aligned}
\left\|A_{M}^{\sigma, 0}\right\|^{\ell}\left\|\left(A_{M}^{\sigma, 0}\right)^{-1}\right\|^{|I|+2 \ell} & =\mathcal{C}(\sigma)^{\ell}\left\|\left(A_{M}^{\sigma, 0}\right)^{-1}\right\|^{|I|+\ell} \\
& \leq C(1+\mathcal{C}(\sigma))^{|J|}\left\|\left(A_{M}^{\sigma, 0}\right)^{-1}\right\|^{|I|}\left(1+\left\|\left(A_{M}^{\sigma, 0}\right)^{-1}\right\|\right)^{|J|} .
\end{aligned}
$$

Proposition 5.7 follows. 
We conclude with the proof of Proposition 2.2:

Proof of Proposition 2.2 From Proposition 3.1

$$
\mathbf{W}_{0}^{V, \sigma, T}=\mathcal{T}^{T}\left(\mathbf{W}_{0}^{T}\right),
$$

where $\mathcal{T}^{T}$ is defined by Eq. 3.5. Let $h_{i}(t)=t Y_{i}, t \in\left[0, T_{i}\right]$. Clearly $\mathcal{T}^{T_{i}}\left(h_{i}\right)=\gamma_{i}$ where $\gamma_{i}$ is as in Eq. 2.7. Hence, from [2, Proposition 3.3.13],

$$
\partial_{\gamma_{i}} \mathbf{W}_{0}^{V, \sigma, T}=\mathcal{T}^{T}\left(\partial_{h_{i}} \mathbf{W}_{0}^{T}\right) .
$$

From Eq. 3.4,

$$
\partial_{h_{i}} \mathbf{W}_{0}^{T}=\left(\bigotimes_{k \neq i} \mathbf{W}_{0}^{T_{k}}\right) \otimes \partial_{h} \mathbf{W}_{0}^{T_{i}},
$$

where $h(t)=t, t \in\left[0, T_{i}\right]$. But the derivatives of the classical Wiener measure over a finite interval are known. It is clear from $[1,10]$ that $h$ belongs to the CameronMartin space $H\left(\mathbf{W}_{0}^{T_{i}, \mathbb{R}}\right)$ and therefore is a differentiable vector. Also

$$
\begin{aligned}
\left(\partial_{h_{i}} \mathbf{W}_{0}^{T_{i}, \mathbb{R}}\right)\left(d \eta_{i}\right) & =-\left(\int_{0}^{T_{i}} h_{i}^{\prime}(u) d \eta_{i}(u)\right) \mathbf{W}_{0}^{T_{i}, \mathbb{R}}\left(d \eta_{i}\right) \\
& =\eta_{i}\left(T_{i}\right) \mathbf{W}_{0}^{T_{i}, \mathbb{R}}\left(d \eta_{i}\right) .
\end{aligned}
$$

Proposition 2.2 follows.

\section{The Derivatives of $P_{t}^{\sigma}(x, y)$}

The goal of this section is the proof of the following result.

Theorem 6.1 There is a $C>0$ such that

$$
\begin{aligned}
\left|X^{I} Y^{J} P_{t}^{\sigma}(x, y)\right| \leq & C(\mathcal{C}(\sigma)+1)^{\varkappa(I, J)}\left\|\left(A_{M}^{\sigma, 0}\right)^{-1}\right\|^{|I|}\left(1+\left\|\left(A_{M}^{\sigma, 0}\right)^{-1}\right\|\right)^{|J|} \\
& \times\left(1+\left\|A_{V}^{\sigma}\right\|\right)^{(\varkappa(I, J)+1) / 2} \\
& \times \mathcal{D}\left(A^{\sigma}\right)\left(1+|(x, y)|_{k_{o}}\right)^{\left(2 \bar{\rho}_{\Xi}+3 k_{o}+1\right)|I|} \\
& \times \exp \left(-\frac{D\|x\|^{2 /\left(k_{o}+1\right)} \phi_{k_{o}}(x)}{\left\|A^{\sigma}\right\|}-\frac{D\|y\|^{2}}{\left\|A_{V}^{\sigma}\right\|}\right) .
\end{aligned}
$$

Proof It is an immediate consequence of Proposition 5.7 and Theorem 5.5 that

$$
\begin{aligned}
\left|\tilde{X}^{I} Y^{J} P_{t}^{\sigma}\right| \leq & C(\mathcal{C}(\sigma)+1)^{\varkappa(I, J)}\left\|\left(A_{M}^{\sigma, 0}\right)^{-1}\right\|^{|I|}\left(1+\left\|\left(A_{M}^{\sigma, 0}\right)^{-1}\right\|\right)^{|J|} \\
& \times(1+\|x\|)^{|I|+2|J|} B_{t}^{\sigma, \varkappa(I, J)}(x, y) \exp \left(-\frac{D\|x\|^{2 /\left(k_{o}+1\right)} \phi_{k}(x)}{\left\|A^{\sigma}\right\|}-\frac{D\|y\|^{2}}{\left\|A_{V}^{\sigma}\right\|}\right) .
\end{aligned}
$$


Of course for $f \in C^{\infty}(N)$,

$$
X_{i} f(x, y)=\sum_{j=1}^{m} c_{i, j}(y) X_{j} f(x, y),
$$

where the operator on the right acts only on $x$ and

$$
\operatorname{Ad}_{y}\left(X_{i}\right)=\sum_{j=1}^{m} c_{i, j}(y) X_{j}
$$

From Eq. 4.3,

$$
\begin{aligned}
\left|X_{i} f(x, y)\right| & \leq C(1+|y|)^{k_{o}} \max _{j}\left|\tilde{X}_{j} f(x, y)\right| \\
& \leq C\left(1+|(x, y)|_{k_{o}}\right)^{k_{o}} \max _{j}\left|\tilde{X}_{j} f(x, y)\right| .
\end{aligned}
$$

More generally,

$$
\left|X^{I} f(x, y)\right| \leq\left(1+|(x, y)|_{k_{o}}\right)^{k_{o}|I|} \max _{|\tilde{I}| \leq|I|} \tilde{X}^{I} f(x, y) \mid .
$$

Also

$$
1+\|x\| \leq\left(1+|(x, y)|_{k_{o}}\right)^{k_{o}+1} .
$$

Theorem 6.1 follows.

\section{The Derivatives of the Poisson Kernel}

From Corollary 2.1 we need to bound $\lim _{t \rightarrow \infty} \mathbf{E}_{a} X^{I} Y^{J} \check{P}_{t}^{\sigma}\left(g_{o}\right)$, where $\left|g_{o}\right|=1$. Theorem 6.1 bounds $X^{I} Y^{J} P_{t}^{\sigma}$ by a function of the exponential functionals $A_{M}^{\sigma, 0}(0, t)$ and $A_{V}^{\sigma}(0, t)$. There is an exact formula for such an expectation for $t=\infty$ in the case of independent Brownian motions. Specifically, let $b(t)=\left(b_{1}(t), \ldots, b_{n}(t)\right)$ be an $n$-dimensional Brownian motion normalized so that $\operatorname{Var} b_{j}(t)=2 t$ and let $\alpha \in \mathbb{R}^{n}$. Let $\tau \in \mathbb{R}^{n}$ satisfy $\tau \cdot \alpha>0$. We define

$$
\begin{aligned}
\sigma^{\tau}(t) & =\tau \cdot(b(t)-2 \alpha t), \\
\Lambda^{\tau} & =\max _{0 \leq u<\infty} \tau \cdot \sigma(u), \\
A^{\tau} & =\int_{0}^{\infty} e^{\tau \cdot \sigma(u)} d u,
\end{aligned}
$$

where $A^{\tau}$ and $\Lambda^{\tau}$ are thought of as random variables on $C\left([0, \infty), \mathbb{R}^{k}\right)$.

Remark Note that our current use use of " $\Lambda^{\tau}$ " and " $A^{\tau}$ " is a change of notation from that of Section 4. 
Let $\left\{e_{1}, \ldots, e_{k}\right\}$ be the standard basis for $\mathbb{R}^{k}$. Then $\left\{e_{1} \cdot b(t), \ldots, e_{k} \cdot b(t)\right\}$ is a family of independent, one-dimensional Brownian motions with $\operatorname{Var}\left(e_{i} \cdot b(t)\right)=2 t$. For $b \in \mathbb{R}^{k}$ and $u \in\left(\mathbb{R}^{+}\right)^{k}$ we define

$$
u^{b}=\prod_{i} u_{i}^{b_{i}}
$$

The following result follows from Theorem 2.2 of [12].

Proposition 7.1 Let $f$ be a continuous function on $\left(\mathbb{R}^{+}\right)^{k}$. Then

$$
\mathbf{E}_{a} f\left(A^{e_{1}}, \ldots, A^{e_{k}}\right)=C e^{2 a \cdot \alpha} \int_{\left(\mathbb{R}^{+}\right)^{k}} f(u) u^{-2 \alpha} \exp \left(-\sum_{i} \frac{e^{a_{i}}}{u_{i}}\right) \frac{d u}{u},
$$

where

$$
\frac{d u}{u}=\prod_{1}^{k} \frac{d u_{i}}{u_{i}}
$$

Remarkably, the function $\tau \mapsto \log \left(A^{\tau}\right)$ behaves in some respects as if it depended linearly on $\tau$. To explain this, let $\mathcal{T}=\left\{\tau_{1}, \ldots, \tau_{\ell}\right\}$ be an orthogonal family of vectors in $\mathbb{R}^{k}$ such that $\alpha \cdot \tau_{i}>0$ for all $i$. For $u=\alpha /\|\alpha\|$ let

$$
d(\mathcal{T})=2^{-\ell+3} \sqrt{2} \min \left\{\frac{u \cdot v}{\|v\|} \mid v=\tau_{i_{1}}+\cdots+\tau_{i_{j}}, 1 \leq i_{j} \leq \ell, 1 \leq j \leq \ell\right\} .
$$

We assume the positivity condition-i.e., the $\lambda_{i, j}$ in Eq. 1.7 are non-negative. Let

$$
d_{i}=d\left(\left\{\lambda_{i, 1} e_{1}, \ldots, \lambda_{i, k} e_{k}\right\} \backslash\{0\}\right), \quad 1 \leq i \leq q .
$$

We let

$$
x_{i}=A^{e_{i}}, 1 \leq i \leq k
$$

The following is a direct consequence of Corollary 7.11 which is proved in Section 7.1 below.

To simplify notation we write $\mathbf{W}_{a}$ instead of $\mathbf{W}_{a}^{\infty, \mathbb{R}}$ to denote the Wiener measure on $C([0,+\infty), \mathbb{R})$.

Proposition 7.2 For all $a \in \mathbb{R}^{n}, n \in \mathbb{Z}$, and $1 \leq i \leq q$,

$$
\mathbf{W}_{a}\left(\left\{e^{n-1} \leq A^{\lambda_{i}}\left(x^{\lambda_{i}}\right)^{-1} \leq e^{n}\right\}\right) \leq C e^{-\frac{1}{2} d\|\alpha\||n|} .
$$

where $x=\left(x_{1}, \ldots, x_{k}\right)$ and $d=\min _{1 \leq i \leq q} d_{i} / 2$.

Theorem 7.3 Suppose that $\|\alpha\|>4 \varkappa / d$ where $\varkappa=\varkappa(I, J)$. Then for $|z|=1$, and for all $a \in \mathbb{R}^{n}$,

$$
\left(X^{I} Y^{J} v\right)\left(a^{-1} z a\right) \leq C e^{a \cdot(\rho+\lambda(I, J))} e^{-a_{\Xi}^{\min }(\varkappa / 2+|I|+|J|)} .
$$


Proof According to Eq. 2.2,

$$
\left(X^{I} Y^{J} v\right)\left(a^{-1} z a\right)=e^{a \cdot(\rho+\lambda(I, J))} \lim _{t \rightarrow \infty} \mathbf{E}_{a} X^{I} Y^{J} P_{t}^{\sigma}(z) .
$$

Let

$$
\begin{aligned}
& \mathcal{X}_{\ell, n}=\left\{\sigma \mid e^{n-\ell} \leq A^{\lambda_{i}}\left(x^{\lambda_{i}}\right)^{-1} \leq e^{n}, \forall 1 \leq i \leq q\right\}, \\
& \mathcal{Y}_{\ell, n}=\mathcal{X}_{\ell, n} \backslash \mathcal{X}_{\ell-1, n-1}, \\
& \mathcal{Z}_{\ell, n}=\mathcal{Y}_{\ell, n} \backslash \mathcal{Y}_{\ell-1, n} .
\end{aligned}
$$

Then, almost certainly,

$$
C\left([0, \infty), \mathbb{R}^{k}\right)=\bigcup_{\ell \in \mathbb{N}, n \in \mathbb{Z}} \mathcal{Z}_{\ell, n}
$$

For $\sigma \in \mathcal{Z}_{\ell, n}$,

$$
\begin{array}{ll}
\forall i, & e^{n-\ell} x^{\lambda_{i}} \leq A^{\lambda_{i}} \leq e^{n} x^{\lambda_{i}}, \\
\exists i, & e^{n-1} \leq A^{\lambda_{i}}\left(x^{\lambda_{i}}\right)^{-1} \leq e^{n}, \\
\exists j, & e^{n-\ell} \leq A^{\lambda_{j}}\left(x^{\lambda_{j}}\right)^{-1} \leq e^{n-\ell+1} .
\end{array}
$$

In particular from Proposition 7.2,

$$
P_{x}\left(\mathcal{Z}_{\ell, n}\right) \leq C \min \left\{e^{-d\|\alpha\||n-\ell|}, e^{-d\|\alpha\||n|}\right\} .
$$

Corollary 7.4 For $\ell \in \mathbb{N}$ and $n \in \mathbb{Z}$,

$$
P_{x}\left(\mathcal{Z}_{\ell, n}\right) \leq C e^{-\frac{d\|\alpha\|}{4}(|n|+\ell)} .
$$

Proof This follows from the observation that for $\ell \in \mathbb{N}$ and $n \in \mathbb{Z}$.

$$
\max \{|n-\ell|,|n|\} \geq(|n|+\ell) / 4 \text {. }
$$

Let

$$
\begin{aligned}
H(\sigma)= & \mathcal{D}\left(A^{\sigma}\right)(\mathcal{C}(\sigma)+1)^{\varkappa}\left\|\left(A_{M}^{\sigma, 0}\right)^{-1}\right\|^{|I|}\left(1+\left\|\left(A_{M}^{\sigma, 0}\right)^{-1}\right\|\right)^{|J|} \\
& \times\left(1+\left\|A_{V}^{\sigma}\right\|\right)^{(\varkappa+1) / 2} \exp \left(-\frac{D^{\prime}}{\left\|A^{\sigma}\right\|}\right),
\end{aligned}
$$

where $D^{\prime}>0$ and $\varkappa=\varkappa(I, J)$. From Theorem 6.1, we need to bound

$$
\mathbf{E}_{a} H \mathbf{1}_{\mathcal{Z}_{\ell, n}} \leq\left(\mathbf{E}_{a} H^{2} \mathbf{1}_{\mathcal{Z}_{\ell, n}}\right)^{1 / 2} P_{a}\left(\mathcal{Z}_{\ell, n}\right)^{1 / 2}
$$

For $x=\left(x_{1}, \ldots, x_{k}\right) \in\left(\mathbb{R}^{+}\right)^{k}$ let

$$
Q_{M}(x)=\sum_{i=1}^{m} x^{\lambda_{i}}, \quad Q_{V}(x)=\sum_{i=m+1}^{q} x^{\lambda_{i}},
$$

and

$$
Q(x)=Q_{M}(x)+Q_{V}(x) .
$$


We also set

$$
x^{-1}=\left(x_{1}^{-1}, \ldots, x_{k}^{-1}\right) .
$$

From Eq. 7.1, for $\sigma \in \mathcal{Z}_{\ell, n}$,

$$
\begin{aligned}
\mathcal{D}\left(A^{\sigma}\right) & \leq C e^{\frac{(\ell-n) \varkappa}{2}} x^{-\frac{\rho}{2}}, \\
\left\|\left(A_{M}^{\sigma, 0}\right)^{-1}\right\| & \leq C e^{\ell-n} Q_{M}\left(x^{-1}\right) \leq C e^{\ell} Q_{M}\left(x^{-1}\right), \\
\left\|A_{V}^{\sigma}\right\| & \leq C e^{n} Q_{V}(x), \\
\left\|A^{\sigma}\right\| & \leq C e^{n} Q(x), \\
\mathcal{C}(\sigma) & \leq C e^{\ell} \max _{1 \leq i, j \leq m} x^{\lambda_{i}} x^{-\lambda_{i}} \leq C e^{\ell} Q_{M}(x) Q_{M}\left(x^{-1}\right) .
\end{aligned}
$$

Hence, for some scalar $C>0$,

$$
\begin{aligned}
H(\sigma)^{2} \mathbf{1}_{\mathcal{Z}_{\ell, n} \leq} \leq & e^{(2 \ell-n) \varkappa+2(\ell-n)|I|+2 \ell|J|}\left(1+e^{n(q+1)}\right) x^{-\rho} \\
& \times\left(Q_{M}(x) Q_{M}\left(x^{-1}\right)+1\right)^{\varkappa} Q_{M}\left(x^{-1}\right)^{2|I|}\left(1+Q_{M}\left(x^{-1}\right)\right)^{2|J|} \\
& \times\left(1+Q_{V}(x)\right)^{\varkappa+1} .
\end{aligned}
$$

Thus

$$
\begin{aligned}
\mathbf{E}_{a} H(\sigma)^{2} \mathbf{1}_{\mathcal{Z}_{\ell, n} \leq} & C e^{2 a \cdot \alpha+n(1-2|I|)+2 \ell(|I|+|J|)} \\
& \times \int x^{-2 \alpha} Q_{M}\left(x^{-1}\right)^{2|I|} R(x) S(x) \exp \left(-\sum_{i} \frac{e^{a_{i}}}{x_{i}}\right) \frac{d x}{x},
\end{aligned}
$$

where

$$
\begin{aligned}
R(x) & =\left(Q_{M}(x)+1\right)^{\varkappa+2|I|}\left(Q_{V}(x)+1\right)^{2|J|} \text { and } \\
S(x) & =\left(Q_{M}\left(x^{-1}\right)+1\right)^{\varkappa+2|J|} .
\end{aligned}
$$

For $x, a \in \mathbb{R}^{k}$ we define

$$
e^{a} x=\left(e^{a_{1}} x_{1}, \ldots, e^{a_{k}} x_{k}\right)
$$

Clearly for $b \in \mathbb{R}^{k}$,

$$
\left(e^{a} x\right)^{b}=e^{a \cdot b} x^{b} .
$$

Hence, for $a \in-A^{+}$,

$$
\begin{aligned}
Q_{M}\left(e^{a} x\right) & \leq e^{a_{\mathrm{m}}^{\max }} Q_{M}(x) \leq Q_{M}(x), \\
Q_{M}\left(\left(e^{a} x\right)^{-1}\right) & \leq e^{-a_{\mathrm{\Xi}}^{\min }} Q_{M}\left(x^{-1}\right), \\
1+Q_{M}\left(\left(e^{a} x\right)^{-1}\right) & \leq e^{-a_{\mathrm{m}}^{\min }}\left(1+Q_{M}\left(x^{-1}\right)\right) .
\end{aligned}
$$

Analogous statements hold for $Q_{V}$.

We make the changes of variables

$$
e^{a} u=x, \quad \frac{d x}{x}=\frac{d u}{u}
$$


followed by $u=x$ obtaining

$$
\begin{aligned}
\mathbf{E}_{a} H(\sigma)^{2} \mathbf{1}_{\mathcal{Z}_{\ell, n} \leq} \leq & e^{n(1-2|I|)+2 \ell(|I|+|J|)} e^{-a_{\mathrm{\Xi}}^{\min }(\varkappa+2|I|+2|J|)} \\
& \times \int x^{-2 \alpha} Q_{M}\left(x^{-1}\right)^{2|I|} R(x) S\left(x^{-1}\right) \exp \left(-\sum_{i} \frac{1}{x_{i}}\right) \frac{d x}{x} .
\end{aligned}
$$

The convergence of this integral is clear for $x_{i}$ small. For large $x$ the growth of the integrand is determined by $R(x)$. But

$$
R(x) \leq C\left(1+\sum_{i=1}^{m} x^{(\varkappa+2|I|) \lambda_{i}}+\sum_{j=m+1}^{q} x^{2|J| \lambda_{j}}\right) .
$$

This integral will be finite provided for $1 \leq \ell \leq k$

$$
\begin{array}{r}
2 \alpha_{\ell}-(\varkappa+2|I|) \lambda_{i, \ell}>0,1 \leq i \leq m \text { and } \\
2 \alpha_{\ell}-2|J| \lambda_{j, \ell}>0, m+1 \leq j \leq q
\end{array}
$$

which is implied by Eq. 1.8.

Then from Corollary 7.4,

$$
\left(\mathbf{E}_{a} H(\sigma)^{2} \mathbf{1}_{\mathcal{Z}_{\ell, n}}\right)^{1 / 2} P_{a}\left(\mathcal{Z}_{\ell, n}\right)^{1 / 2} \leq C e^{-a_{\Xi}^{\min }(\varkappa / 2+|I|+|J|)} e^{n(1-2|I|)+2 \ell(|I|+|J|)} e^{-\frac{d\|\alpha\|}{4}(|n|+\ell)} .
$$

Assumption (1.8) also implies

$$
\frac{d\|\alpha\|}{4}>\max \{2(|I|+|J|), 1\} .
$$

Summing over $\ell$ and $n$ shows that

$$
\mathbf{E}_{a} H(\sigma) \leq C e^{-a_{\Xi}^{\min }(\varkappa / 2+|I|+|J|)} .
$$

Our theorem follows.

7.1 Reduction to Independence

Theorem 2.17 on p. 135 of [8] implies the following three results.

Theorem 7.5 Let $\tau \in \mathbb{R}^{k}$. For all $D>0$ there is a $C_{D}$ such that for all $n \in \mathbb{Z}$,

$$
\mathbf{W}_{0}\left(\left\{e^{n-1} \leq A^{\tau} e^{-\Lambda^{\tau}} \leq e^{n}\right\}\right) \leq C_{D} e^{-D|n|} .
$$

It is easily seen that this implies:

Corollary 7.6 Let $\tau \in \mathbb{R}^{k}$. Given $d$, D there is a $C_{D}$ such that for all $n \in \mathbb{N}$,

$$
\mathbf{W}_{0}\left(\left\{n-1 \leq\left|\log \left(A^{\tau}\right)-\Lambda^{\tau}\right| \leq n\right\}\right) \leq C_{D} e^{-D n} .
$$

Since

$$
\left\{n \leq\left|\log \left(A^{\sigma}\right)-\Lambda^{\sigma}\right|\right\}=\bigcup_{k=1}^{\infty}\left\{n+k-1 \leq\left|\log \left(A^{\sigma}\right)-\Lambda^{\sigma}\right| \leq n+k\right\}
$$


we see that:

Corollary 7.7 Let $\tau \in \mathbb{R}^{k}$. Given $D$ there is a $C_{D}$ such that for all $n \in \mathbb{N}$,

$$
\mathbf{W}_{0}\left(\left\{n \leq\left|\log \left(A^{\tau}\right)-\Lambda^{\tau}\right|\right\}\right) \leq C_{D} e^{-D n} .
$$

Let $\tau_{1}, \tau_{2} \in \mathbb{R}^{n}$ be orthogonal - i.e., $\tau_{1} \cdot \tau_{2}=0$. Assume that $\tau_{i} \cdot \alpha=\alpha_{i}>0$. Then $\sigma^{i}(t)=\tau_{i} \cdot \sigma_{t}$ is a pair of independent Brownian motions with drifts $-2 \alpha_{i}$ and

$$
\operatorname{Var}\left(\sigma^{i}(t)\right)=2 t\left\|\tau_{i}\right\|^{2}
$$

Let $\Lambda^{i}=\Lambda^{\tau_{i}}$ and $\Lambda=\Lambda^{\tau_{1}+\tau_{2}}$. For $u=\alpha /\|\alpha\|$, let

$$
d=d\left(\left\{\tau_{1}, \tau_{2}\right\}\right)=2 \sqrt{2} \min \left\{\frac{u \cdot \tau_{1}}{\left\|\tau_{1}\right\|}, \frac{u \cdot \tau_{2}}{\left\|\tau_{2}\right\|}, \frac{u \cdot\left(\tau_{1}+\tau_{2}\right)}{\left\|\tau_{1}+\tau_{2}\right\|}\right\}
$$

Proposition 7.8 For $d$ as in Eq. 7.2,

$$
\mathbf{W}_{0}\left(\left\{n \leq\left|\Lambda-\left(\Lambda^{1}+\Lambda^{2}\right)\right|\right\}\right) \leq C e^{-\|\alpha\| d n} .
$$

In the proof we will need the following very well known result (see e.g. [3] on p. 197).

Lemma 7.9 Let $w(t)$ be the one dimensional Brownian motion with negative drift, i.e., $w(t)=b(t)-\gamma t$ with $\gamma>0$. Then

$$
\mathbf{W}_{0}\left(\sup _{t \geq 0} w(t) \geq k\right) \leq e^{-c k},
$$

where

$$
c=2 \gamma / \sqrt{\operatorname{Var} w(1)} \text {. }
$$

Proof of Proposition 7.8 In order to prove Eq. 7.3 it is enough to show that

$$
\mathbf{W}_{0}\left(\left\{n \leq\left|\Lambda-\left(\Lambda^{1}+\Lambda^{2}\right)\right| \leq n+1\right\}\right) \leq C e^{-\|\alpha\| d n}
$$

with a constant $C>0$ not depending on $n$.

First we note that the probability in Eq. 7.4 is bounded by

$$
\mathbf{W}_{0}\left(n \leq \Lambda-\left(\Lambda^{1}+\Lambda^{2}\right) \leq n+1\right)+\mathbf{W}_{0}\left(-n-1 \leq \Lambda-\left(\Lambda^{1}+\Lambda^{2}\right) \leq-n\right) .
$$

Now we estimate the first probability in Eq. 7.5.

$$
\begin{aligned}
\mathbf{W}_{0}(n & \left.\leq \Lambda-\left(\Lambda^{1}+\Lambda^{2}\right) \leq n+1\right) \\
& =\sum_{k=1}^{\infty} \mathbf{W}_{0}\left(n-\Lambda \leq-\left(\Lambda^{1}+\Lambda^{2}\right) \leq n+1-\Lambda \text { and } k-1<\Lambda \leq k\right) \\
& \leq \sum_{k=1}^{\infty} \mathbf{W}_{0}\left(n-k \leq-\left(\Lambda^{1}+\Lambda^{2}\right) \leq n+2-k \text { and } k-1<\Lambda \leq k\right) .
\end{aligned}
$$


By the Cauchy-Schwarz inequality the above series is dominated by

$$
\sum_{k=1}^{\infty} \mathbf{W}_{0}\left(-n-2+k \leq \Lambda^{1}+\Lambda^{2} \leq-n+k\right)^{1 / 2} \mathbf{W}_{0}(k-1<\Lambda)^{1 / 2}
$$

Notice that since our process starts from 0 the first $n$ terms (for $k \leq n$ ) in the above series are zero.

Furthermore, by Lemma 7.9, $\mathbf{W}_{0}(k<\Lambda) \leq e^{-c k}$, where $c=\sqrt{2}\left(\tau_{1}+\tau_{2}\right) \cdot \alpha / \| \tau_{1}+$ $\tau_{2} \|$. Hence, the first probability on the right in Eq. 7.5, can be estimated by

$$
\begin{aligned}
& \sum_{k=n+1}^{\infty} \mathbf{W}_{0}\left(-n-2+k \leq \Lambda^{1}+\Lambda^{2} \leq-n+k\right)^{1 / 2} e^{-c(k-1) / 2} \\
& =\sum_{l=-1}^{\infty} \mathbf{W}_{0}\left(l \leq \Lambda^{1}+\Lambda^{2} \leq l+2\right)^{1 / 2} e^{-c(l+n+1) / 2} \\
& \leq e^{-c n / 2} \sum_{l=-1}^{\infty} e^{-c(l+1) / 2} \leq C e^{-c n / 2} .
\end{aligned}
$$

Similarly, the second probability in Eq. 7.5 can be estimated by

$$
\begin{gathered}
\sum_{k=1}^{\infty} \mathbf{W}_{0}\left(n+k-1 \leq \Lambda^{1}+\Lambda^{2}\right)^{1 / 2} \mathbf{W}_{0}(k-1<\Lambda \leq k)^{1 / 2} \\
\leq \sum_{k=1}^{\infty} \mathbf{W}_{0}\left((n+k-1) / 2 \leq \Lambda^{1}\right)^{1 / 2} e^{-c(k-1) / 2} \\
\quad+\sum_{k=1}^{\infty} \mathbf{W}_{0}\left((n+k-1) / 2 \leq \Lambda^{2}\right)^{1 / 2} e^{-c(k-1) / 2} .
\end{gathered}
$$

By Lemma 7.9, $\mathbf{W}_{0}\left((n+k-1) / 2 \leq \Lambda^{i}\right)^{1 / 2} \leq e^{-c_{i}(n+k-1) / 4}$, where $c_{i}=\sqrt{2} \tau_{i} \cdot \alpha /\left\|\tau_{i}\right\|$. Hence the second probability in Eq. 7.5 is dominated by $C_{1} e^{-c_{1} n / 4}+C_{2} e^{-c_{2} n / 4}$.

Let $\Lambda^{i}=\Lambda^{\tau_{i}}$ and $\Lambda=\Lambda^{\tau}$ where $\tau=\sum_{1}^{\ell} \tau_{i}$.

Corollary 7.10 For $n \in \mathbb{N}$,

$$
\mathbf{W}_{0}\left(\left\{n \leq\left|\Lambda-\sum_{i=1}^{\ell} \Lambda^{i}\right|\right\}\right) \leq C e^{d\left(\left\{\tau_{1}, \ldots, \tau_{\ell}\right\}\right)\|\alpha\| n} .
$$

Proof We reason by induction on $\ell$. The $\ell=1$ case is trivial and Proposition 7.8 is the $\ell=2$ case so assume that $\ell \geq 3$. Let

$$
\begin{gathered}
\tilde{\tau}_{2}=\sum_{i=2}^{\ell} \tau_{i}, \\
\tilde{\Lambda}^{2}=\Lambda^{\tilde{\tau}_{2}} .
\end{gathered}
$$


From Proposition 7.8 and induction

$$
\begin{aligned}
& \mathbf{W}_{0}\left(\left\{n \leq\left|\Lambda-\left(\Lambda^{1}+\tilde{\Lambda}^{2}\right)\right|\right\}\right) \leq C e^{-d\left(\left\{\tau_{1}, \tilde{\tau}_{2}\right\}\right)\|\alpha\| n} \\
& \mathbf{W}_{0}\left(\left\{n \leq\left|\tilde{\Lambda}_{2}-\sum_{i \geq 2} \Lambda^{i}\right|\right\}\right) \leq C e^{-d\left(\left\{\tau_{2}, \ldots, \tau_{\ell}\right\}\right)\|\alpha\| n}
\end{aligned}
$$

Clearly

$$
\frac{1}{2} \min \left\{d\left(\left\{\tau_{1}, \tilde{\tau}_{2}\right\}\right), d\left(\left\{\tau_{2}, \ldots, \tau_{\ell}\right\}\right)\right\} \geq d\left(\left\{\tau_{1}, \ldots, \tau_{\ell}\right\}\right)
$$

Also

$$
\Lambda-\sum_{i=1}^{\ell} \Lambda^{i}=\left(\Lambda-\left(\Lambda^{1}+\tilde{\Lambda}^{2}\right)\right)+\left(\tilde{\Lambda}_{2}-\sum_{i=2}^{\ell} \Lambda^{i}\right)
$$

Hence

$$
\left\{n \leq\left|\Lambda-\sum_{i=1}^{\ell} \Lambda^{i}\right|\right\} \subset\left\{\frac{n}{2} \leq\left|\Lambda-\left(\Lambda^{1}+\tilde{\Lambda}^{2}\right)\right|\right\} \cup\left\{\frac{n}{2} \leq\left|\tilde{\Lambda}_{2}-\sum_{i=2}^{\ell} \Lambda^{i}\right|\right\}
$$

from which the corollary follows.

Corollary 7.11 Let $\tau=\sum_{1}^{\ell} \tau_{i}$ and let $e_{i}=\tau_{i} /\left\|\tau_{i}\right\|$. For $a \in \mathbb{R}^{k}$ and $n \in \mathbb{Z}$,

$$
\mathbf{W}_{a}\left(\left\{e^{n-1} \leq A^{\tau}\left(\prod_{1}^{\ell}\left(A^{e_{i}}\right)^{\left\|\tau_{i}\right\|}\right)^{-1} \leq e^{n}\right\}\right) \leq C e^{-\frac{1}{2} d\left(\left\{\tau_{1}, \ldots, \tau_{\ell}\right\}\right)\|\alpha\||n|}
$$

Proof We may assume $a=0$ since the probability of the stated set is clearly independent of the starting point. If $\tau$ belongs to the set described in the left side of the preceding inequality then

$$
|n|-1 \leq\left|\log A^{\tau}-\sum_{1}^{\ell}\right| \tau_{i}\left|\log A^{e_{i}}\right|
$$

\section{Of course}

$$
\begin{aligned}
& \log A^{\tau}-\sum_{1}^{\ell}\left|\tau_{i}\right| \log A^{e_{i}} \\
& =\left(\Lambda^{\tau}-\sum_{1}^{\ell} \Lambda^{\tau_{i}}\right)+\left(\log A^{\tau}-\Lambda^{\tau}\right)+\left(\sum_{1}^{\ell}\left|\tau_{i}\right| \Lambda^{e_{i}}-\left|\tau_{i}\right| \log A^{e_{i}}\right) \\
& \equiv B+C+D
\end{aligned}
$$


Assume $|n|>1$. Then

$$
\begin{aligned}
|n|-1 \leq|B+C+D| \Rightarrow\left(|B| \geq \frac{|n|-1}{2}\right) \text { or }\left(|C+D| \geq \frac{|n|-1}{2}\right) \\
\Rightarrow\left(|B| \geq \frac{|n|-1}{2}\right) \text { or }\left(|C| \geq \frac{|n|-1}{4}\right) \\
\quad \text { or }\left(\exists i,\left|\tau_{i}\right|\left|\Lambda^{\tau_{i}}-\log A^{\tau_{i}}\right| \geq \frac{|n|-1}{4 \ell}\right) .
\end{aligned}
$$

Our corollary follows from Corollaries 7.7 and 7.10.

Open Access This article is distributed under the terms of the Creative Commons Attribution License which permits any use, distribution, and reproduction in any medium, provided the original author(s) and the source are credited.

\section{References}

1. Bogachev, V.I.: Gaussian Measures. Mathematical Surveys and Monographs, vol. 62. American Mathematical Society, Providence, RI (1998)

2. Bogachev, V.I.: Differentiable Measures and the Malliavin Calculus. Mathematical Surveys and Monographs, vol. 164. American Mathematical Society, Providence, RI (2010)

3. Borodin, A.N., Salminen, P.: Handbook of Brownian Motion-Facts and Formulae, 2nd edn. Probability and its Applications. Birkhäuser Verlag, Basel (2002)

4. Buraczewski, D., Damek, E., Hulanicki, A.: Asymptotic behavior of Poisson kernels on NA groups. Comm. Partial Differential Equations 31(10-12), 1547-1589 (2006)

5. Damek, E.: Left-invariant degenerate elliptic operators on semidirect extensions of homogeneous groups. Stud. Math. 89, 169-196 (1988)

6. Damek, E., Hulanicki, A.: Asymptotic behavior of the invariant measure for a diffusion related to an $N A$ group. Colloq. Math. 104(2), 285-309 (2006)

7. Damek, E., Hulanicki, A., Urban, R.: Martin boundary for homogeneous riemannian manifolds of negative curature at the bottom of the spectrum. Rev. Mat. Iberoamericana 17(2), 257-293 (2001)

8. Damek, E., Hulanicki, A., Zienkiewicz, J.: Estimates for the Poisson kernels and their derivatives on rank one $N A$ groups. Stud. Math. 126(2), 115-148 (1997)

9. Folland, G.B., Stein, E.: Hardy Spaces on Homogeneous Groups. Princeton University Press (1982)

10. Norin, N.V.: The extended stochastic integral in linear spaces with differentiable measures and related topics. World Scientific Publishing Co., Inc., River Edge, NJ (1996)

11. Penney, R., Urban, R.: Estimates for the Poisson kernel and the evolution kernel on the Heisenberg group. J. Evol. Equ. 12(2), 327-351 (2012)

12. Penney, R., Urban, R.: Estimates for the Poisson kernel and the evolution kernel on nilpotent meta-abelian groups. Studia Math. (to appear). arXiv:math.FA/1108.2515

13. Urban, R.: Estimates for the derivatives of the Poisson kernels on homogeneous manifolds of negative curvature. Math. Z. 240(4), 745-766 (2002) 